\title{
Mipomersen: Pharmacology, Clinical Trials and Its Potential Role in Therapy
}

\author{
Jeffery Evans", Dorothy Ann Shelton \\ University of Louisiana at Monroe College of Pharmacy \\ *Corresponding Author: jevans@ulm.edu
}

Copyright $(2013$ Horizon Research Publishing All rights reserved.

\begin{abstract}
Mipomersen is a novel pharmacologic agent that utilizes anti-sense technology to reduce LDL in patients with very high LDL. The medication has been approved in the United States based on a series of small trials proving efficacy even with when added to traditional therapy. Safety concerns have been raised due to a potential for liver toxicity.
\end{abstract}

Keywords Mipomersen, Anti-sense, Hyperlipidemia,

Lipids

\section{Introduction}

HMG-CoA reductase inhibitors (statins) are considered the most effective medications for the treatment of hyperlipidemia. ${ }^{1}$ They reduce morbidity and mortality for both primary and secondary prevention in coronary heart disease patients. These agents are usually recommended first line, however in patients that do not reach low-density lipoprotein cholesterol (LDL-C) goals with these medications additional agents are needed. This is more common in patients who already have coronary artery disease or need a greater reduction in LDL-C then a statin can provide. Bile acid sequesterants are effective at lowering LDL-C cholesterol however have significant medication interactions. ${ }^{2}$ Fibrates and niacin may also be used to reduce LDL-C cholesterol, but have the largest effect on High Density Lipoprotien cholesterol (HDL-C) and triglycerides. ${ }^{1}$ There are doubts about ezetimibe, since the ENHANCE trial $^{3}$ has had doubts about whether it was only effective at lowering LDL-C versus actually reducing the risk of heart disease. However, even when all of these medications are utilized, some patients are either intolerant to adverse effects of the medication or they do not reach LDL goals (very prevalent in patients with familial hypercholesterolemia.)

Dyslipidemia is a common disease that traditionally impacts adults. In the case of familial hypercholesterolemia an individual has high circulating levels of total cholesterol and LDL-C even at young ages. Familial hypercholesterolemia is an autosomal dominant genetic disorder resulting from a single genetic mutation on chromosome 19 in the LDL receptor (LDL-R). ${ }^{4}$ This mutation prevents LDL-C from binding to its receptors in the liver, which further prevents the uptake and degradation of LDL-C. There are two types of familial hypercholesterolemia, homozygous and heterozygous. Homozygous familial hypercholesterolemia is less common, but more severe because the individual has inherited two mutated alleles and has no functional LDL receptors which leads to significantly high levels of LDL. Heterozygous familial hypercholesterolemia is more common, but less severe because the individual has inherited one mutated allele and one normal allele and have about one-half the number of functioning LDL receptors when compared to an individual without familial hypercholesterolemia.

Mipomersen is one of a few agents currently in the pipeline for both genetic and non-genetic causes of hypercholesterolemia. Others include proprotien convertase subtilisen/kexin type 9 (PCSK9) inhibitors ${ }^{5,6}$, with REGN727/SAR236553 leading the way, help patients that have intact LDL-C receptors lower cholesterol by significant percentages even when added to full dose atorvastatin. The medication is currently moving into large, phase three trials ${ }^{6}$. Another class of medication is the microsomal triglyceride transfer protein inhibitors, an example molecule is lomitapide. ${ }^{5}$ Lomitapide was approved for use in patients with homozygous familial hypercholesterolemia in December 2012 in the United States. It is projected to cost between 200,000 and 300,000 dollars per year. ${ }^{7}$

\section{Pharmacology and Kinetics of Mipomersen}

Mipomersen mechanism of action is through a process of anti-sense binding. It seeks out ribonucleic acid (RNA) responsible for coding for Apolipoprotien B100 (ApoB100) and binds to it. ${ }^{8}$ Once mipomersen binds, it attracts ribonuclease H1 (RNase H1) that cleaves the RNA and makes the entire molecule useless to the body. This process then frees the mipomersen molecule to bind to another strand 
of $\mathrm{RNA}^{8}$ Without the proper coding to make ApoB100 molecules, the production of LDL-C cholesterol is reduced and the molecules that are reduced may have a lower atherosclerotic predisposition. ApoB100 serves as the core to the structure of LDL-C and of lipoprotein (a) (LpA)which are both risk factors for heart disease. Both of these molecules are produced in the liver and then enter the circulatory system where they may eventually deposit into the lining of the arteries.

Mipomersen is considered a second generation anti-sense oligonucleotide. ${ }^{8}$ Second generation molecules surpass first generation molecules by increasing the affinity the molecule has for its target RNA. Changes are made at both the 5' and 3' ends by adding a 2' methoxyenthyl substituted nucleotide, which increases the ability of the molecule to bind to the target region of the RNA and also reduces the ability of natural nucleases to break down the molecule. ${ }^{8}$ This will extend the half-life of the molecule potentially by weeks. There also appears to be a reduction in the pro-inflammatory effects of the molecule with the addition of the 2 ' methoxyenthyl group.

Due to the molecular structure of mipomersen, it is not able to be given orally and must be given as an injection. ${ }^{9}$ Subcutaneous injections results in about $83 \%$ of the dose being absorbed into systemic circulation. Mean peak time for the medications was between 3.4 and 4.0 hours. Once in the plasma, a rapid distribution phase occurs where the medication is deposited in the tissues of the kidneys, lymph nodes, liver, spleen, and thyroid/parathyroid. ${ }^{8}$ The medication then undergoes a prolonged elimination phase that can last several days. ${ }^{10}$ A mean terminal elimination half-life was shown to be between 46 and 48 days, thus allowing for less frequent dosing of the medication ${ }^{10}$ Dose finding studies found that higher doses of the medication led to higher peak and trough concentrations of the medication.

\section{Clinical Trials}

Mipomersen, an apolipoprotein B synthesis inhibitor, for lowering of LDL-C cholesterol concentrations in patients with homozygous familial hypercholesterolaemia: a randomised, double-blind, placebo-controlled trial.

Raal et $\mathrm{al}^{11}$ studied mipomersen in a population of patients with homozygous familial hypercholesterolemia who were already on maximum tolerated HMG-CoA reductase inhibitors and other lipid lowering medications. The trial was a placebo controlled, double blind, randomized phase 3 trial conducted at several different sites in different countries. Inclusion criteria included a diagnosis of homozygous familial hypercholesterolemia and currently on therapy but not at the LDL-C goal for the patient. The patient had to be at least 12 years old and could have a history of heart disease as long as any cardiac event had occurred more than 12 weeks before the patient was screened for the trial. Additionally, both diet and medication therapy had to be stable prior to and during the trial. Patients were randomized to either receive a placebo injected subcutaneously or $200 \mathrm{mg}$ of mipomersen (160 mg if weight less than $50 \mathrm{~kg}$ ) once weekly for 26 weeks. All patients underwent magnetic resonance imaging (MRI) to determine liver fat composition prior to beginning the trial and a repeat MRI was completed if patients experienced elevated liver function tests during the trial.

A total of 51 patients were randomized to either mipomersen $(n=24)$ or placebo $(n=17$.) Patients receiving mipomersen had an average reduction of LDL-C of $25 \%$ while the placebo group had a $3 \%$ reduction. Patients in the mipomersen group also had statistically significant reductions in ApoB, total cholesterol, non-HDL cholesterol, LpA triglycerides, and very low density lipoprotein (VLDL) and a significant increase in HDL-C cholesterol (15\%.) The medication did not have a significant effect on apo A1.

No serious adverse events that were linked to the medication occurred during the trial. The most common adverse event was injection site reactions ( $76 \%$ mipomersen vs. $24 \%$ placebo) and influenza like symptoms $(29 \%$ mipomersen vs. $24 \%$ placebo.) Patients with mild elevations in AST were similar between the two groups $(35 \%$ mipomersen vs. $41 \%$ placebo) but patients with significant elevations, between three and eight times the upper limit of normal, occurred more frequently in the mipomersen group ( $12 \%$ mipomersen vs. $0 \%$ placebo.) One of the four patients with significant elevations in aspartate aminotransferase (AST) showed an increase in hepatic fat $(9.6 \%$ baseline to $24.8 \%$ at time of elevated enzymes,) two patients did not show a change, and one patient stopped the study drug and did not complete a MRI. This trial begins to establish the limited role for mipomersen in the treatment of homozygous familial hypercholesterolemia. The trial shows effectiveness in both the short term and mid-term periods. Potential issues that arose from the trial were injection site reactions and the increase of liver enzymes three to eight times the upper limit of normal. It is interesting that a link between elevated liver enzymes and an increase in fat content of the liver was not seen.

Potent reduction of apolipoprotein B and low-density lipoprotein cholesterol by short-term administration of an antisense inhibitor of apolipoprotein B.

Kastelein et al ${ }^{12}$ studied mipomersen in patients with only mild hyperlipidemia to determine the safety and effectiveness in this population. Patients were first divided into two separate groups, one that received a single dose of the mipomersen and then after a four week period started on variable dosing of the medication or were just started on the variable dosing of the medication for four weeks. Each of the arms had a controlled placebo group. Patients in the single dose group received either 50,100, 200, $400 \mathrm{mg}$ of mipomersen or a placebo in a buffered saline solution as a single subcutaneous injection. Patients were then followed for four weeks to determine changes in their lipid panels and to evaluate safety outcomes. After four weeks, both groups received a series of three intravenous injections to bolus the liver levels of the medication up to about $70 \%$ of the predicted steady state levels. Patients then received three 
once weekly subcutaneous doses of mipomersen ranging from $50-400 \mathrm{mg}$ or placebo. After the three injections, patients were followed for up to 12 weeks or until their cholesterol levels had returned to at least $90 \%$ of the original value.

Patients receiving the $200 \mathrm{mg}$ dose showed an LDL reduction of $30.6 \%$ at day 25 (four days after the final injection) and continued to show a reductions of about $34 \%$ at day 55 (five weeks after the final injection.) ${ }^{12}$ Patients in the $400 \mathrm{mg}$ group saw a reduction of $43.7 \%$ and $40.7 \%$ reduction in LDL on days 25 and 55 . The 50 and $100 \mathrm{mg}$ doses did not show a statistical difference in LDL or ApoB levels on any day. Both 200 and $400 \mathrm{mg}$ doses significantly reduced ApoB100 levels at day 25 (50.2 and 43.8) and at day 55 (42.4 and 47.4.) These data show a long post medication effect that could indicate very infrequent dosing of the medication.

This trial was completed in adults without any other medical conditions than mild hyperlipidemia and a total cholesterol of less than $300 \mathrm{mg} / \mathrm{dl}$. Twenty-one of 29 patients receiving mipomersen complained of an injection site reaction compared to none of the patients in the placebo group. Four of the 29 patients receiving mipomersen developed elevated liver enzymes and one of the four developed liver enzymes greater than 3 times upper limit of normal.

A different healthier population of patients was chosen for this study compared to the first study. The study was well designed and showed that a population with normal levels of LDL-C would still see a significant reduction in cholesterol when started with the mipomersen. As in the first study, the most frequent side effect was an injection site reaction. Though the lower doses of medication were not shown to lower LDL-C or ApoB, it is interesting that even after 55 days without therapy patients receiving the $200 \mathrm{mg}$ or 400 mg still had significantly lower LDL-C and ApoB levels compared to baseline levels.

Apolipoprotein B synthesis inhibition with mipomersen in heterozygous familial hypercholesterolemia: results of a randomized, double-blind, placebo-controlled trial to assess efficacy and safety as add-on therapy in patients with coronary artery disease.

Continuing the study of mipomersen in patients with genetic abnormalities, Stein et $\mathrm{al}^{13}$ studied the medication in patients with heterozygous familial hypercholesterolemia and heart disease on maximum doses of HMG-COA reductase inhibitors. The trial was a phase 3 multicenter, randomized, double blind, placebo controlled trial.

Patients were 18 years or older with either genetic confirmation of a LDL defect or clinical diagnosis of Heterozygous Familial Hypercholesterolemia (HeFH.) Additionally patients had to have stable coronary artery disease. Patients had to be taking a maximally tolerated dose of a HMG-COA and could be taking other lipid lowering medications. Patients were excluded if their heart disease was not stable, if they had known hepatic disease, or had their cardiovascular medications recently changed.
The trial enrolled 124 patients and randomized 41 patients to placebo and 83 patients to mipomersen $200 \mathrm{mg}$ once weekly. Patients that completed the trial entered into a 24 week follow up without medication or into a open label extension study. Patients receiving mipomersen had an LDL decrease of $28 \%$ during the trial, which was statiscally significant. Additionally, compared to baseline, the mipomersen group saw decreases in ApoB, total cholesterol, non-HDL cholesterol , Lp(A), triglycerides, VLDL, and ApoA1 which were statiscally significant. Neither HDL-C or high sensitivity $\mathrm{C}$-reactive protein changed with mipomersen therapy.

The most prevalent adverse event of injection site reactions occurred in $93 \%$ of the patients receiving mipomersen and $42 \%$ in the placebo group. Other adverse drug events that occurred at a rate of greater than $10 \%$ in the mipomersen group were influenza like symptoms (49.4\%), Nausea (16.9\%), headache (18.1\%), and diarrhea, nasopharyngitis, and cough which occurred at a rate of $10.8 \%$. Elevated liver enzymes were detected in 1 patient in the placebo group and 12 patients in the mipomersen group. Five of the 12 patients with elevated liver enzymes still had elevated enzymes at least one week after the initial findings. As shown in other trials ${ }^{12}$, patients that had elevated levels of enzymes had the largest LDL reductions. Finally, patients receiving mipomersen were found to have a higher level of fat in the liver compared to those receiving placebo. ${ }^{13}$ Patients with higher levels of liver fat had a corresponding increase in alanine aminotransferase (ALT) and AST.

This trial increases the total number of patients exposed to the drug, but otherwise does not add much to what is known about the drug. It is concerning that it seems that there is some link between the effectiveness of the medication in lowering LDL-C and the potential side effects of increased liver fat and elevated liver enzymes.

Randomized, placebo-controlled trial of mipomersen in patients with severe hypercholesterolemia receiving maximally tolerated lipid-lowering therapy.

A final trial looked at adding mipomersen to other drug regimens that included full dose HMG-CoA reductase inhibitors and ezetimibe in some patients. ${ }^{14}$ This trial enrolled 58 patients with 45 patients finishing 26 weeks of therapy of mipomersen $200 \mathrm{mg}$ once weekly subcutaneous injections. During the trial more than $75 \%$ of patients receiving mipomersen saw at least a $15 \%$ decrease in LDL-C compared to only $17 \%$ of patients in the placebo group. Additionally, $25 \%$ of patients in the mipomersen group saw a greater than $50 \%$ reduction in LDL-C. This trial showed a higher average reduction of LDL-C in females (44\%) compared to males $(27 \%)$, but both sexes experienced statistically and clinically significant reductions. Lipoprotien A was also studied and was reduced in the mipomersen group by $33 \%$ compared to the placebo group which had a $1.5 \%$ reduction. HDL-C cholesterol did increase in both groups, but was not significantly different in either group. Triglycerides decreased by $8.6 \%$ in the mipomersen group which was statistically different than the placebo group 
which saw an increase in triglycerides during the study period.

All patients in the mipomersen arm experienced a side effect during the clinical trial. Injection site reactions were the most common adverse event with $79 \%$ of patients in the mipomersen group reporting the side effect. Flu like symptoms were reported in $46 \%$ of patients in the mipomersen group compared to $21 \%$ in the placebo group. All six serious adverse events that occurred during the trial occurred in patients using mipomersen. Only two of the six were considered medication related by the investigators (one patient due to elevated liver enzymes with hepatic steatosis and the other with a stroke and angina.) Six patients of the 39 withdrew from the mipomersen arm prematurely (two of the six met protocol reasons for withdraw). All patients received an MRI prior to the trial to determine liver fat content and those that had significant liver issues during the trial received a repeat MRI to determine changes in liver fat content. Seven of the 12 patients that received a follow up MRI showed elevated amounts of liver fat compared to baseline.

The final trial presented mimics previous trials with the medication surpassing placebo therapy. This trial added in patients who were already on other lipid medications and showed that mipomersen's safety and effectiveness. It is concerning that during the trial 49 patients of the 58 patients enrolled in the trial had a protocol deviation. The most common reason was inadequate time on the study drug. Of these 49 patients, only 16 patients were excluded from the final analysis.

\section{Potential Role in Therapy}

Kynamro (mipomersen, Genzyme) was approved by the United States Food and Drug Administration (US FDA) for patients with homozygous familial hypercholesterolemia on January $29^{\text {th }}, 2013^{3,15}$. The medication received approval as an orphan drug, meaning it receives extended patent life since it is used to treat a rare, life threatening disease. For approval though, the FDA required several longer term studies to be completed including follow-up for antibody development, cancer risk, and hepatic disease risks. Prescribers wanting to prescribe the medication will need to be trained to prescribe prior to being allowed to prescribe the medication. The concerns the US FDA had for mipomersen were echoed by the Committee for Medicinal Products for Human Use (CHMP) of the European Medicines Agency when they recommended against approval of mipomersen in the European Union due to the concerns about cancer and hepatic damage ${ }^{15}$.

Currently the role of mipomersen in non-familial hypercholesterolemia is unknown. It is not recommended, nor should it be used due to a lack of efficacy and safety data, in patients with only hypercholesterolemia. In patients with homozygous familial hypercholesterolemia, mipomersen has a role but only after the patient has been put on other medications including a HMG-CoA reductase inhibitor (preferably titrated to full dose,) ezetimibe, and/or bile acid sequesterants. Due to the significant and warranted safety concerns with the medications, many prescribers may choose the risk of higher rates of heart disease then prescribe this medication to patients. With the recent FDA approval, patients with heterozygous familial hypercholesterolemia may have access to mipomersen, though depending upon the prescribing requirements may be locked out of access to the medication.

\section{Conclusion}

With the release of mipomersen, the treatment plans for the vast majority of patients with hypercholesterolemia will remain the same. Potentially with future research the safety concerns of mipomersen could be reduced, however it seems unlikely that they will go away. With other agents potentially coming to market, some with safety concerns of their own, the usefulness of mipomersen will probably decline even further.

\section{REFERENCES}

[1] Windler E, Zyriax BC, Bamberger C, Rinninger F, Beil FU. Current strategies and recent advances in the therapy of hypercholesterolemia. Atheroscler Suppl 2009;10(5):1-4.

[2] Third Report of the National Cholesterol Education Program (NCEP) Expert Panel on Detection, Evaluation, and Treatment of High Blood Cholesterol in Adults (Adult Treatment Panel III) Final Report. Circulation 2002;106(25):3143.

[3] Kastelein JJ, Akdim F, Stroes ES et al. Simvastatin with or without ezetimibe in familial hypercholesterolemia. N Engl J Med 2008;358(14):1431-1443.

[4] Palacio CH, Harring TR, Nguyen NT, Goss JA, O'Mahony CA. Homozygous familial hypercholesterolemia: case series and review of the literature. Case Rep Transplant. 2011;2011 Epub 2012 Jan 11.

[5] Bell DA, Hooper AJ, Watts GF, Burnett JR. Mipomersen and other therapies for the treatment of severe familial hypercholesterolemia. Vasc Health Risk Manag 2012;8:651-659.

[6] Stein EA, Gipe D, Bergeron J et al. Effect of a monoclonal antibody to PCSK9, REGN727/SAR236553, to reduce low-density lipoprotein cholesterol in patients with heterozygous familial hypercholesterolaemia on stable statin dose with or without ezetimibe therapy: a phase 2 randomised controlled trial. Lancet 2012;380(9836):29-36.

[7] Forbes. FDA Approves Lomitapide For Homozygous Familial Hypercholesterolemia. 12-24-2012. Accessed on 1-30-2013.

[8] Crooke ST, Geary RS. Clinical pharmacological properties of mipomersen (Kynamro), a second generation antisense inhibitor of apolipoprotein B. Br J Clin Pharmacol 2012. 
[9] Thomas T, Ginsberg H. Development of apolipoprotein B antisense molecules as a therapy for hyperlipidemia. Curr Atheroscler Rep 2010;12(1):58-65.

[10] Akdim F, Tribble DL, Flaim JD et al. Efficacy of apolipoprotein B synthesis inhibition in subjects with mild-to-moderate hyperlipidaemia. Eur Heart J 2011;32(21):2650-2659.

[11] Raal FJ, Santos RD, Blom DJ et al. Mipomersen, an apolipoprotein B synthesis inhibitor, for lowering of LDL cholesterol concentrations in patients with homozygous familial hypercholesterolaemia: a randomised, double-blind, placebo-controlled trial. Lancet 2010;375(9719):998-1006.

[12] Kastelein JJ, Wedel MK, Baker BF et al. Potent reduction of apolipoprotein B and low-density lipoprotein cholesterol by short-term administration of an antisense inhibitor of apolipoprotein B. Circulation 2006;114(16):1729-1735.
[13] Stein EA, Dufour R, Gagne C et al. Apolipoprotein B synthesis inhibition with mipomersen in heterozygous familial hypercholesterolemia: results of a randomized, double-blind, placebo-controlled trial to assess efficacy and safety as add-on therapy in patients with coronary artery disease. Circulation 2012;126(19):2283-2292.

[14] McGowan MP, Tardif JC, Ceska R et al. Randomized, placebo-controlled trial of mipomersen in patients with severe hypercholesterolemia receiving maximally tolerated lipid-lowering therapy. PLoS One 2012;7(11):e49006.

[15] FDA approves new orphan drug Kynamro to treat inherited cholesterol disorder. 1-29-2013. 1-30-2013. Ref Type: Online Source

[16] European Medicines Agency. Refusal of the marketing authorisation for Kynamro. 12-13-2012. 1-30-2013. Ref Type: Online Source. 